\title{
Stent placement for benign esophageal leaks, perforations, and fistulae: a clinical prediction rule for successful leakage control
}

Authors

Emo E. van Halsema ${ }^{1}$, Wouter F. W. Kappelle ${ }^{2}$, Bas L. A. M. Weusten ${ }^{3}$, Robert Lindeboom ${ }^{4}$, Mark I. van Berge Henegouwen ${ }^{5}$, Paul Fockens ${ }^{1}$, Frank P. Vleggaar ${ }^{2}$, Manon C. W. Spaander ${ }^{6}$, Jeanin E. van Hooft ${ }^{1}$

Institutions

1 Department of Gastroenterology and Hepatology, Academic Medical Center, Amsterdam, the Netherlands

2 Department of Gastroenterology and Hepatology, University Medical Center Utrecht, the Netherlands

3 Department of Gastroenterology and Hepatology, St Antonius Hospital, Nieuwegein, the Netherlands

4 Department of Clinical Epidemiology and Biostatistics, Academic Medical Center, Amsterdam, the Netherlands

5 Department of Surgery, Academic Medical Center, Amsterdam, the Netherlands

6 Department of Gastroenterology and Hepatology, Erasmus University Medical Center, Rotterdam, the Netherlands

submitted 26.4.2017

accepted after revision 26.7.2017

\section{Bibliography}

DOI https://doi.org/10.1055/s-0043-118591

Published online: 21.9.2017 | Endoscopy 2018; 50: 98-108

(c) Georg Thieme Verlag KG Stuttgart · New York

ISSN 0013-726X

Corresponding author

Jeanin E. van Hooft, MD, PhD, Department of

Gastroenterology and Hepatology, Room C2-115,

Academic Medical Center, Meibergdreef 9, 1105 AZ

Amsterdam, The Netherlands

Fax: +31-20-6917033

j.e.vanhooft@amc.uva.nl

丹 Supplementary material

Online content viewable at:

https://doi.org/10.1055/s-0043-118591
\# Scan this QR-Code for the author's interview.

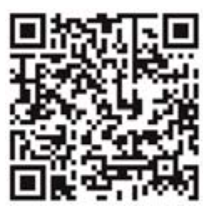

\section{ABSTRACT}

Background and study aims Sealing esophageal leaks by stent placement allows healing in $44 \%-94 \%$ of patients. We aimed to develop a prediction rule to predict the chance of successful stent therapy.

Patients and methods In this multicenter retrospective cohort study, patients with benign upper gastrointestinal leakage treated with stent placement were included. We used logistic regression analysis including four known clinical predictors of stent therapy outcome. The model performance to predict successful stent therapy was evaluated in an independent validation sample.

Results We included etiology, location, C-reactive protein, and size of the leak as clinical predictors. The model was estimated from 145 patients (derivation sample), and 59 patients were included in the validation sample. Stent therapy was successful in $55.9 \%$ and $67.8 \%$ of cases, respectively. The predicted probability of successful stent therapy was significantly higher in success patients compared with failure patients in both the derivation $(P<0.001)$ and validation $(P<0.001)$ samples. The area under the receiver operating characteristic curve was $74.1 \%$ in the derivation sample and $84.7 \%$ in the validation sample. When the model predicted $\geq 70 \%$ chance of success, the positive predictive value was $79 \%$ in the derivation sample and $87 \%$ in the validation sample. When the model predicted $\leq 50 \%$ chance of success, the negative predictive value was $64 \%$ and $86 \%$, respectively.

Conclusions This prediction rule, consisting of four clinical predictors, could identify patients with esophageal leaks who were likely to benefit from or fail on stent therapy. The prediction rule can support clinical decision-making when the predicted probability of success is $\geq 70 \%$ or $\leq 50 \%$. 


\section{Introduction}

Anastomotic leaks after esophagectomy cause severe morbidity, prolongation of hospitalization, intensive care unit (ICU) admission, and even mortality [1-5]. Leakage develops in 6\%$24 \%$ of patients undergoing esophageal surgery with esophagogastrostomy [2,6-13], and leads to increased postoperative mortality rates of $7.2 \%-18.2 \%$ compared with $3.1 \%-6.2 \%$ in patients without leakage [1-3]. The aim of treatment of esophageal leaks is to prevent sepsis and allow healing by administration of antibiotics, bypassing the esophageal defect with a duodenal feeding tube, and adequate drainage of infected fluid collections.

Another way to prevent further contamination of the paraesophageal cavity is by sealing off the defect with an esophageal stent [14]. Endoscopic stent placement for esophageal leaks and perforations allows complete healing in approximately $80 \%$ of cases in pooled literature analyses $[14,15]$. Either fully covered or partially covered self-expandable stents are used for sealing esophageal disruptions. The pitfall of esophageal stent placement is the high risk of stent migration, which occurs in $11 \%-27 \%$ of cases, depending on the type of stent $[14,15]$. Inadequate sealing may be one of the reasons why the clinical success rates of esophageal stent placement vary widely from $44 \%$ to $94 \%$ [15]. Optimal drainage of adjacent fluid collections is also essential for the success of stent therapy. Other factors that contribute to successful stent therapy are the size of the defect, the etiology of the leak, the severity of sepsis, and the delay between diagnosis and stent insertion [16-20].

Leakage control is crucial for the healing process in these severely ill patients. If we were able to predict the success of stent therapy, we could select patients who are likely to benefit from stent placement. In this way, patients in whom stent therapy is likely to fail can be identified and treated differently. Therefore, the aim of the current study was to evaluate the performance of a clinical prediction rule to predict the chance of successful stent therapy in the treatment of benign upper gastrointestinal leaks.

\section{Patients and methods}

This study was designed as a multicenter retrospective cohort study including three tertiary care university hospitals and one general teaching hospital from the Netherlands. The study was reviewed by the Medical Ethics Review Committee of the Academic Medical Center and did not apply to the Dutch Act "Medical Research Involving Human Subjects” (date of review: 17 June 2015).

We electronically searched the endoscopic database ENDOBASE (Olympus Medical Systems, Hamburg, Germany) and a prospectively collected database of all esophagectomy patients to identify suitable candidates for inclusion. All patients who received an esophageal stent for the treatment of benign anastomotic leakage, perforation or fistula were included. Patients with active malignant esophageal disease were excluded from the analysis. The details of patient selection are presented in - Fig. 1 .
A leak or perforation was diagnosed based on the combination of clinical signs and radiological or endoscopic evidence of a transmural esophageal defect. Symptomatology included at least one of the following symptoms: severe cervical or thoracic pain, subcutaneous emphysema, elevated C-reactive protein (CRP) level or leukocyte count, sepsis, air leakage through the drain or wound, and/or excessive drain or wound production. Radiological evidence included leakage of oral contrast outside the esophagus at computed tomography (CT) scan or esophagogram. A fistula was defined as a radiologically or endoscopically proven connection between the esophagus and the respiratory tract, the cutis or the pleura. Perforations included all iatrogenic and spontaneous transmural esophageal lacerations.

The primary outcome of the study was successful leakage control after esophageal stent placement. We defined success as no need for additional invasive procedures (surgery or percutaneous drainage) after stent removal without persisting symptoms of leakage, or as successful sealing without further symptoms of leakage in case it was decided that the stent was left in permanently. Follow-up was calculated as the time between the first stent placement procedure and the date of last contact.

\section{Procedures}

Because of the retrospective study design, procedures were not standardized. In general, when an upper gastrointestinal leak was suspected, imaging studies (CT scan or esophagogram) and/or endoscopy were performed to confirm the diagnosis. In case of a suspected cervical anastomotic leak, the neck wound was re-opened for exploration and drainage. Patients received antibiotics and were kept on a "nil by mouth" regimen with duodenal tube feeding or feeding jejunostomy. Infected fluid collections were drained by radiological tube placement whenever possible.

The treating surgeon and gastroenterologist determined whether stent placement was indicated. Proximal stent placement across the upper esophageal sphincter was considered a contraindication for stent placement. Stent placement was performed endoscopically at the endoscopy ward with or without fluoroscopic guidance. In case of ICU admission, endoscopic stent placement took place at the ICU without the support of fluoroscopy. Anesthesia during the procedure consisted of monitored anesthesia care using propofol or conscious sedation using midazolam and/or fentanyl. Either fully covered or partially covered self-expandable metal stents (SEMSs) were used, which were preferably removed after 4-6 weeks. Stents were removed by pulling either the proximal end or distal end (inside-out) of the stent with a grasping forceps. The stent-instent technique was used for the removal of embedded partially covered SEMSs [21].

The aim of stent therapy was to achieve leakage control, defined as a stable clinical condition, allowing oral intake and healing of the defect. When stent dysfunction and persistent leakage was suspected, endoscopy was performed. In case of stent migration or inadequate sealing, stents were either repositioned or replaced. Once leakage control had been achieved, patients were discharged from the hospital, and returned to the outpatient clinic for stent removal and further ambulatory care. 


\section{AMC \\ - Endobase search for esophageal stents \\ - Period: \\ Jan 2004 - Jul 2015 \\ - Cases identified: N = 439 \\ Excluded: \\ - Gastric outlet obstruction: $\mathrm{N}=71$ \\ - Malignant indications: $\mathrm{N}=223$ \\ - Benign strictures: $\mathrm{N}=53$}

Stents for benign esophageal leaks, perforations and fistulae: $\mathrm{N}=92$

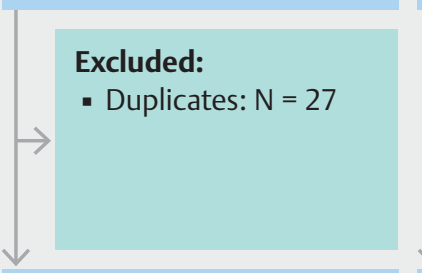

Unique patients who received an esophageal stent for leakage: $\mathrm{N}=65$

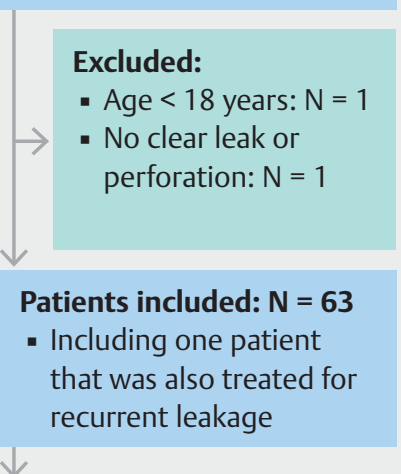

Derivation sample: $\mathbf{N}=145$

\section{AZN}

- Surgical database of esophagectomy

- Period:

Jan 2011 - Jul 2016

- Unique patients identified: $\mathrm{N}=162$

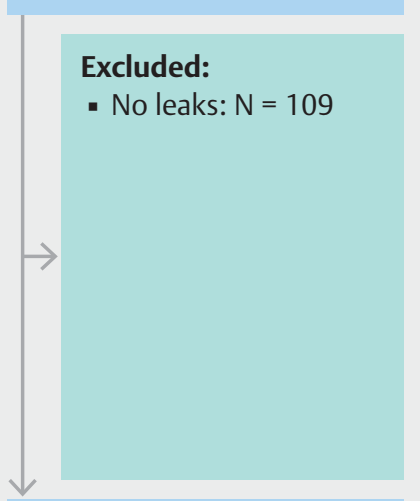

Esophageal leaks: $\mathrm{N}=53$

Excluded:

- Benign stricture: $\mathrm{N}=1$

- No stent: $\mathrm{N}=17$

- Duplicate: $N=1$

\section{Unique patients who} received an esophageal stent for leakage: $\mathrm{N}=34$

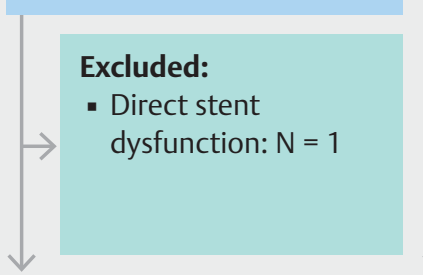

\section{Patients included: $\mathbf{N}=\mathbf{3 3}$}

\section{Patients included: $\mathbf{N}=\mathbf{4 7}$ \\ - Including one patient that was also treated for recurrent leakage}

\section{EMC}

- Endobase search for esophageal stents for leaks, perforations, fistulae

- Period: Jan 2010 - Jul 2016

- Unique patients

\begin{tabular}{|l} 
Excluded: \\
- Gastric outlet \\
obstruction: $\mathrm{N}=14$ \\
- Malignant \\
indications: $\mathrm{N}=39$ \\
- Benign strictures: \\
$\mathrm{N}=9$ \\
- Other indications: \\
$\mathrm{N}=4$ \\
- No stent: $\mathrm{N}=30$ \\
\end{tabular}

Unique patients who received an esophageal stent for leakage: $\mathrm{N}=62$

\section{Excluded:}

- Age < 18 years: $\mathrm{N}=3$

- Insufficient data:

$\mathrm{N}=4$

- Aortoesophageal fistula: $N=1$

- No clear leak or perforation: $\mathrm{N}=4$

- Direct stent

- Direct surgery after stent: $\mathrm{N}=1$ identified: $\mathrm{N}=158$ migration: $\mathrm{N}=2$

\section{UMCU}

- Endobase search for esophageal stents

- Period:

Jan 2010 - Apr 2016

- Cases identified: N = 346

\section{Excluded:}

- Duplicates: $\mathrm{N}=113$

- Gastric outlet obstruction: $\mathrm{N}=25$

- Malignant indications: $\mathrm{N}=88$

- Benign strictures: $\mathrm{N}=41$

- Other indications:

$\mathrm{N}=1$

- No stent: $\mathrm{N}=23$

Patients with stents for benign esophageal leaks, perforations and fistulae: $\mathrm{N}=55$

Patients added
manually: $\mathrm{N}=9$
Unique patients who
received an esophageal
stent for leakage: $\mathrm{N}=64$

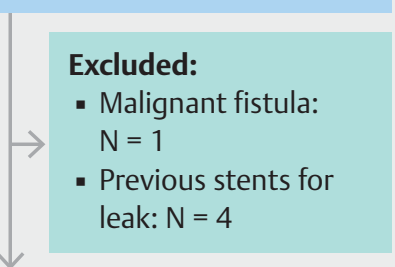

Patients included: $\mathbf{N}=\mathbf{5 9}$

Validation sample: $\mathbf{N}=\mathbf{5 9}$

- Fig. 1 Flow chart of patient selection. AMC, Academic Medical Center; AZN, St Antonius Hospital; EMC, Erasmus University Medical Center; UMCU, University Medical Center Utrecht. 
Table 1 Population characteristics.

Derivation sample

$n=145$

Hospital, n (\%)

- Academic Medical Center Amsterdam

- Erasmus University Medical Center Rotterdam

- St Antonius Hospital Nieuwegein

- University Medical Center Utrecht

Male sex, n (\%)

Age, mean $\pm S D$, years

Etiology, n (\%)

- Anastomotic leak

- Perforation

- Fistula

History of esophageal cancer, yes, n (\%)

History of thoracic/cervical radiotherapy, yes, n (\%)

History of upper gastrointestinal surgery

$$
\text { - No }
$$

- Esophagectomy

- Gastrectomy

- Bariatric surgery

- Other

- Unknown

Location of defect, n (\%)

- Proximal esophagus (<25 cm from incisors)

- Mid esophagus (25-30 cm from incisors)

- Distal esophagus (>30 cm from incisors)

C-reactive protein level, median (IQR), mg/L

Leukocyte count; median (IQR), 109/ L

Size of defect, n (\%)

$$
\text { - }<1 \mathrm{~cm}
$$

- $1-2 \mathrm{~cm}$

. $>2 \mathrm{~cm}$

Previous leak-related treatment, $\mathrm{n}$ (\%)

\section{- None}

- Drainage by tube placement

- Surgical interventions

- Endoscopic interventions

Delay until stent placement, median (IQR), days

Stents used, $\mathrm{n}$

- FCSEMS, n (\%)

- PCSEMS, n (\%)

\begin{tabular}{|c|c|}
\hline $64(44.1)$ & $0(0)$ \\
\hline $48(33.1)$ & $0(0)$ \\
\hline $33(22.8)$ & $0(0)$ \\
\hline $0(0)$ & $59(100)$ \\
\hline $105(72.4)$ & $38(64.4)$ \\
\hline $63.9 \pm 10.6$ & $63.2 \pm 10.6$ \\
\hline
\end{tabular}

\begin{tabular}{|c|c|}
\hline $72(49.7)$ & $35(59.3)$ \\
\hline $48(33.1)$ & $19(32.2)$ \\
\hline $25(17.2)$ & $5(8.5)$ \\
\hline $90(62.1)$ & $39(66.1)$ \\
\hline $79(54.5)$ & $19(32.2)$ \\
\hline
\end{tabular}

\begin{tabular}{|c|c|}
\hline $49(33.8)$ & $18(30.5)$ \\
\hline $86(59.3)$ & $24(40.7)$ \\
\hline $4(2.8)$ & $14(23.7)$ \\
\hline $4(2.8)$ & $0(0)$ \\
\hline $2(1.4)$ & $2(3.4)$ \\
\hline $0(0)$ & $1(1.7)$ \\
\hline
\end{tabular}

73 (50.3)

$24(40.7)$

23 (15.9)

10 (16.9)

$49(33.8)$

$25(42.4)$

$165(89-306)$

$275(158-400)$

$14.3(10.6-20.9)$

$18.4(11.9-24.9)$

68 (46.9)

21 (35.6)

44 (30.3)

21 (35.6)

$33(22.8)$

$17(28.8)$

61 (42.1)

25 (42.4)

68 (46.9)

24 (40.7)

$11(7.6)$

$10(16.9)$

$5(3.4)$

0 (0)

$1(0-7)$

$0(0-3)$

301

93

$174(57.8)$

17 (18.3)

$117(38.9)$ 
- Table 1 (Continuation)

\begin{tabular}{|c|c|c|}
\hline & $\begin{array}{l}\text { Derivation sample } \\
n=145\end{array}$ & $\begin{array}{l}\text { Validation sample } \\
n=59\end{array}$ \\
\hline - Other, n (\%) & $1(0.3)$ & $0(0)$ \\
\hline - Stent type unknown, n (\%) & $9(3.0)$ & $0(0)$ \\
\hline Patients receiving multiple stents, n (\%) & $63(43.4)$ & $18(30.5)$ \\
\hline - No. of stents per patient, median (IQR) & $2(2-3)$ & $2(2-3)$ \\
\hline Leak-related ICU admission, n (\%) & $93(64.1)$ & $32(54.2)$ \\
\hline - Duration, median (IQR), days & $12(3-25)$ & $11(7-23)$ \\
\hline Successful stent therapy, yes, n (\%)) & $81(55.9)$ & $40(67.8)$ \\
\hline
\end{tabular}

\section{Data collection}

The variables presented at baseline ( $\downarrow$ Table 1 ) were collected from the electronic medical records at the participating hospitals. CRP level $(\mathrm{mg} / \mathrm{L})$ and leukocyte count $\left(10^{9} / \mathrm{L}\right)$ were defined as highest value in the 7 days before the first stent placement procedure.

To determine the defect size, we collected the endoscopic images of the leaks at the time of the baseline procedure. The images were independently assessed by four experienced interventional endoscopists (B.W., F.V., M.S., and J.v.H.). They could choose between three categories: $<1 \mathrm{~cm}, 1-2 \mathrm{~cm}$, and $>2 \mathrm{~cm}$. Assessment of the defect size by all four raters was possible in $63 \%$ of cases (129/204), with complete agreement in $27 \%$ (35/129) and three out of four agreement in $42 \%$ (54/129). The interobserver agreement was fair (intraclass correlation two-way random single measures model 0.54). For analysis, we chose the category that received the majority of votes. When there was no majority or the endoscopic images were missing, we used the estimation from the endoscopy report whenever available.

We further retrieved the data on previous upper gastrointestinal surgery, synchronous esophageal strictures, stent type and size, esophageal contrast studies, further leakage-related treatments after stent placement, time and method of stent removal, adverse events, ICU admission, recurrence of esophageal cancer, and mortality.

\section{Statistical analysis}

The prediction model was estimated using the data of $145 \mathrm{pa}$ tients from the Academic Medical Center, Erasmus Medical Center, and St Antonius Hospital (“derivation sample”). The patient cohort from the University Medical Center Utrecht $(n=59)$ was collected independently by W.K. and F.V. and was therefore used for external validation of the model. We selected four known predictors based on face validity and the literature:

1. etiology of the leak, categorized as: 1) anastomotic dehiscence, 2) iatrogenic/spontaneous perforation, and 3) esophago-respiratory, -cutaneous or -pleural fistula;

2. location of the leak, categorized as: 1) proximal esophagus $<25 \mathrm{~cm}$ from the incisors, 2) mid esophagus $25-30 \mathrm{~cm}$ from the incisors, and 3) distal esophagus $>30 \mathrm{~cm}$ from the incisors;

3. CRP level $(\mathrm{mg} / \mathrm{L})$, defined as highest value in the 7 days before the first stent placement procedure;

4. size of the leak as estimated during endoscopy, categorized as: 1$)<1 \mathrm{~cm}, 2) 1-2 \mathrm{~cm}$, and 3) $>2 \mathrm{~cm}$.

In the derivation sample, missing values included CRP level $(\mathrm{n}=$ $35 ; 24 \%)$, leukocyte count $(n=34 ; 23 \%)$, defect size $(n=22$; $15 \%)$, and delay until stent placement $(n=3 ; 2 \%)$. In the validation sample, missing values included history of esophageal cancer $(n=3 ; 5 \%)$, previous leak-related treatment $(n=2 ; 3 \%)$, history of radiotherapy $(n=2 ; 3 \%)$, CRP level ( $n=14 ; 24 \%)$, leukocyte count ( $n=13 ; 22 \%)$, size of defect $(n=12 ; 20 \%)$, and delay until stent placement $(n=2 ; 3 \%)$. For descriptive purposes, missing data were imputed using single stochastic imputation to obtain plausible values for missing units reflecting the variation present in the dataset. The logistic regression estimates of the four-variable prediction model were obtained by pooling the coefficients of five rounds of imputations based on 12 predictor variables including the outcome variable [22]. Linearity in the logit of the continuous variable "CRP" was checked by restricted cubic spline indicating no violation of the assumption. From the log odds of successful stent therapy, derived from the regression equation, we calculated the probability of successful stent therapy for each patient. Discriminative performance was evaluated with Nagelkerke's R2 and receiver operating characteristic - area under the curve (ROC-AUC), and fit of the model with the Hosmer-Lemeshow test based on four risk strata. To estimate the optimism of the model, we performed bootstrap validation using 300 bootstrap samples and applied uniform shrinkage on the model coefficients to correct for optimism [23]. We used the Utrecht cohort (UMCU, > Fig.1) for external validation of the model. We calculated the chance of successful stent therapy for each patient using the prediction model after shrinkage. To evaluate the performance of the model in the external cohort, we again calculated Nagelkerke's R2 test, ROCAUC, and Hosmer-Lemeshow test based on three risk strata.

Continuous variables with a normal distribution are presented as mean with standard deviation, and skewed data as median 
Table 2 Failure of stent therapy.

\begin{tabular}{|l|c|c|}
\hline Reasons for failure of stent therapy & $\begin{array}{l}\text { Derivation sample } \\
\mathbf{n}=\mathbf{6 4}\end{array}$ & $\begin{array}{l}\text { Validation sample } \\
\mathbf{n}=19\end{array}$ \\
\hline Surgical closure/reconstruction & $19(29.7)$ & $\mathbf{3}(15.8)$ \\
\hline Death because of uncontrolled leakage and ongoing sepsis & $14(21.9)$ & $0(0)$ \\
\hline Readmission for persisting symptomatic leakage & $7(10.9)$ & $1(5.3)$ \\
\hline Diverting cervical esophagostomy & $6(9.4)$ & $0(0)$ \\
\hline Additional tube drainage & $6(9.4)$ & $0(0)$ \\
\hline Additional endoscopic interventions & $4(6.3)$ & $2(10.5)$ \\
\hline Death because of major bleed with stent in situ & $3(4.7)$ & $2(10.5)$ \\
\hline Death with symptomatic leakage in the presence of recurrent malignancy & $3(4.7)$ \\
\hline Additional surgical cleansing & $1(1.6)$ \\
\hline Death for unknown reasons with stent in situ and symptomatic leakage & $1(1.6)$ \\
\hline Patients who died during follow-up & $37(57.8)$ \\
\hline - Death related to persisting leakage & $23(35.9)$ \\
\hline - Death unrelated to leakage & $9(10.5)$ \\
\hline - Relationship unknown & $5(0)$ \\
\hline
\end{tabular}

with interquartile range (IQR). We considered two-sided $P$ values $<0.05$ to be statistically significant. For statistical analyses we used RStudio: Integrated Development for R, Version 3.3.2 (RStudio Inc., Boston, Massachusetts, USA) using the mice (multiple imputation) and rms (regression modeling) packages.

\section{Results}

\section{Patient characteristics}

The derivation sample consisted of 143 unique patients who underwent endoscopic stent placement for upper gastrointestinal leakage ( $>$ Fig.1). Two further patients were also treated for a recurrent leak that developed more than 1 year after closure of the primary defect, so we included a total of 145 cases in the analysis. Patient characteristics are presented in - Table 1. In the derivation sample, fully covered SEMSs (FCSEMSs) and partially covered SEMSs (PCSEMSs) remained in situ for a median time of 33 days (IQR $13-57$ days) and 32 days (IQR $11-46$ days), respectively $(P=0.26)$. Stents were finally removed in 121 patients (83.4\%), 21 patients (14.5\%) died with the stent in situ, 2 patients (1.4\%) were still alive with the stent in place at the end of follow-up, and removal status was unknown in one patient $(0.7 \%)$. The median follow-up period in the derivation sample was 316 days (IQR $97-742$ days). In the validation sample $(n=59)$, the median time that PCSEMSs and FCSEMSs were in situ was 23 days (IQR 12-28 days) and 35 days (IQR 24-87 days), respectively $(P=0.001)$. Stents were finally removed in 47 patients (79.7\%) and 12 patients (20.3\%) died with the stent in situ. The median follow-up period in the validation sample was 143 days (IQR 40-692 days). The success rate of stent therapy was $55.9 \%$ in the derivation sample and $67.8 \%$ in the validation sample. See $>$ Table 2 for the failure cases and - Table 3 for success rates within different subgroups.

\section{Clinical predictors}

The prediction rule predicted the probability of successful stent therapy for individual patients based on four clinical predictors: 1) etiology of the leak, 2) location of the leak, 3) baseline CRP level, and 4) size of the leak. In the multivariable logistic regression model, etiology fistulae (estimated regression coefficient2.219, $P=0.001$ ) and defect size $>2 \mathrm{~cm}$ (estimated regression coefficient $-1.174, P=0.02$ ) were statistically significant predictors of an adverse outcome of stent therapy ( $\triangleright$ Table 4 ). The prediction rule formula after shrinkage is available online as "Supplementary Material." The individual probability of success can be derived from the nomogram ( $\triangleright$ Fig. 2 ).

\section{Performance of the prediction rule}

In the derivation sample, the median predicted probability of success was $62.6 \%$ (range $3.4 \%-88.7 \%$ ). Patients who actually failed on stent therapy had a median predicted probability of success of $48.3 \%$ (range $3.4 \%-86.0 \%$ ) compared with $66.9 \%$ (range $14.7 \%-88.7 \%$ ) in patients in whom stent therapy was actually successful $(P<0.001)$. The prediction rule explained $26.1 \%$ of the variance in outcome (Nagelkerke's R2). The overall discriminative accuracy of the model was $74.1 \%$ (ROC-AUC). Calibration of the model was good with at most $11.6 \%$ absolute difference between the actually observed risks and predicted risks (Hosmer-Lemeshow, chi-squared 1.19, df2, $P=0.55$ ) ( Fig.3a). After correction for optimism by shrinkage of the model, the median predicted probability of success was $59.7 \%$ (range $9.7 \%-86.5 \%$ ) in the validation sample. The median predicted probability of success was $43.9 \%$ (range $9.7 \%-$ 
Table 3 Success rates of stent therapy within different subgroups.

\begin{tabular}{|c|c|c|c|c|}
\hline & \multicolumn{2}{|c|}{ Derivation sample $(n=145)$} & \multicolumn{2}{|c|}{ Validation sample $(n=59)$} \\
\hline & Success $(n=81)$ & Failure $(n=64)$ & Success $(n=40)$ & Failure $(n=19)$ \\
\hline \multicolumn{5}{|l|}{ Sex, n (\%) } \\
\hline - Male & $58(55.2)$ & $47(44.8)$ & $23(60.5)$ & $15(39.5)$ \\
\hline - Female & $23(57.5)$ & $17(42.5)$ & $17(81.0)$ & $4(19.0)$ \\
\hline Age, mean $\pm S D$, years & $64.3 \pm 11.1$ & $63.4 \pm 9.8$ & $61.4 \pm 11.3$ & $67.2 \pm 7.7$ \\
\hline \multicolumn{5}{|l|}{ Etiology, n (\%) } \\
\hline - Anastomotic leak & $41(56.9)$ & $31(43.1)$ & $25(71.4)$ & $10(28.6)$ \\
\hline - Perforation & $36(75.0)$ & $12(25.0)$ & $15(78.9)$ & $4(21.1)$ \\
\hline - Fistula & $4(16.0)$ & $21(84.0)$ & $0(0)$ & $5(100)$ \\
\hline \multicolumn{5}{|l|}{ History of esophageal cancer, n (\%) } \\
\hline - No & $35(63.6)$ & $20(36.4)$ & $15(75.0)$ & $5(25.0)$ \\
\hline - Yes & $46(51.1)$ & $44(48.9)$ & $25(64.1)$ & $14(35.9)$ \\
\hline \multicolumn{5}{|l|}{ History of thoracic/cervical radiotherapy, n (\%) } \\
\hline - No & $43(65.2)$ & $23(34.8)$ & $29(72.5)$ & $11(27.5)$ \\
\hline - Yes & $38(48.1)$ & $41(51.9)$ & $11(57.9)$ & $8(42.1)$ \\
\hline \multicolumn{5}{|l|}{ Location of defect, n (\%) } \\
\hline - Proximal esophagus & $39(53.4)$ & $34(46.6)$ & $17(70.8)$ & $7(29.2)$ \\
\hline - Mid esophagus & $8(34.8)$ & $15(65.2)$ & $5(50.0)$ & $5(50.0)$ \\
\hline - Distal esophagus & $34(69.4)$ & $15(30.6)$ & $18(72.0)$ & $7(28.0)$ \\
\hline C-reactive protein level, median (IQR), mg/L & $165(81-293)$ & $162(92-315)$ & $274(154-390)$ & $276(195-400)$ \\
\hline Leukocyte count, median (IQR), $10^{9} / \mathrm{L}$ & $15.0(11.3-22.0)$ & $13.0(10.3-20.8)$ & $14.9(11.8-25.0)$ & $19.9(13.6-23.8)$ \\
\hline \multicolumn{5}{|l|}{ Size of defect, n (\%) } \\
\hline . $<1 \mathrm{~cm}$ & $41(60.3)$ & $27(39.7)$ & $15(71.4)$ & $6(28.6)$ \\
\hline . $1-2 \mathrm{~cm}$ & $25(56.8)$ & $19(43.2)$ & $18(85.7)$ & $3(14.3)$ \\
\hline . $>2 \mathrm{~cm}$ & $15(45.5)$ & $18(54.5)$ & $7(41.2)$ & $10(58.8)$ \\
\hline Delay until stent placement, median (IQR), days & $1(0-3)$ & $1(0-13)$ & $0(0-2)$ & $2(0-6)$ \\
\hline
\end{tabular}

$79.1 \%$ ) for patients who actually failed and $65.7 \%$ (range 46.7 $\%-86.5 \%$ ) for patients in whom stent therapy was actually successful $(P<0.001)$. Nagelkerke's R2 was $45.2 \%$ and the ROC - AUC was $84.7 \%$. The difference between the actually observed and predicted risks was at most 39.3\% (Hosmer-Lemeshow, chi-squared 9.46, df1, $P=0.002$ ) ( $>$ Fig.3b).

\section{Classification accuracy}

The positive predictive value for a predicted probability cutoff of $\geq 70 \%$ was $79 \%(38 / 48)$ in the derivation sample and $87 \%$ $(13 / 15)$ in the validation sample. The false positive rate using that cutoff was $16 \%(10 / 64)$ and $11 \%(2 / 19)$, respectively. The negative predictive value for a predicted probability cutoff of $\leq$ $50 \%$ was $64 \%(32 / 50)$ in the derivation sample and $86 \%(12 / 14)$ in the validation sample. The false negative rate using that cutoff was $22 \%(18 / 81)$ and $5 \%$ (2/40), respectively (> Table 5 ).

\section{Discussion}

We developed a novel prediction rule, consisting of four clinical variables, to predict the probability of successful stent therapy for individual patients with benign upper gastrointestinal leaks. The etiology, location, CRP level, and size of the leak together significantly discriminated between failure and success of stent therapy, including in an independent sample of patients from a different center. External validation showed that patients with a predicted probability of $\geq 70 \%$ had an $87 \%$ chance of success and patients with a predicted probability of $\leq 50 \%$ had an $86 \%$ chance of failure. Using these cutoff values, the prediction rule can be a tool to guide physicians in clinical decision-making and informing patients.

We used a pre-specified model with four clinical predictors that we selected based on face validity and the literature. The 
- Table 4 Outcomes of multivariable logistic regression analysis in the derivation sample.

\begin{tabular}{|c|c|c|c|c|}
\hline & Estimated coefficient & SE & $95 \% \mathrm{Cl}$ & $P$ value \\
\hline \multicolumn{5}{|l|}{ Etiology ${ }^{1}$} \\
\hline - Perforation & 0.702 & 0.593 & $-0.473,1.878$ & 0.24 \\
\hline - Fistula & -2.219 & 0.670 & $-3.548,-0.890$ & 0.001 \\
\hline \multicolumn{5}{|l|}{ Location of defect ${ }^{2}$} \\
\hline - Mid esophagus & -0.825 & 0.601 & $-2.015,0.365$ & 0.17 \\
\hline - Distal esophagus & -0.129 & 0.571 & $-1.258,1.000$ & 0.82 \\
\hline CRP level & -0.003 & 0.002 & $-0.007,0.002$ & 0.21 \\
\hline \multicolumn{5}{|l|}{ Size of defect ${ }^{3}$} \\
\hline . $1-2 \mathrm{~cm}$ & -0.567 & 0.469 & $-1.495,0.361$ & 0.23 \\
\hline . $>2 \mathrm{~cm}$ & -1.174 & 0.506 & $-2.176,-0.171$ & 0.02 \\
\hline \multicolumn{5}{|c|}{$\begin{array}{l}\mathrm{Cl} \text {, confidence interval; CRP, C-reactive protein. } \\
1 \text { Reference category anastomotic dehiscence. } \\
{ }^{2} \text { Reference category proximal esophagus. } \\
{ }^{3} \text { Reference category }<1 \mathrm{~cm} \text {. }\end{array}$} \\
\hline
\end{tabular}

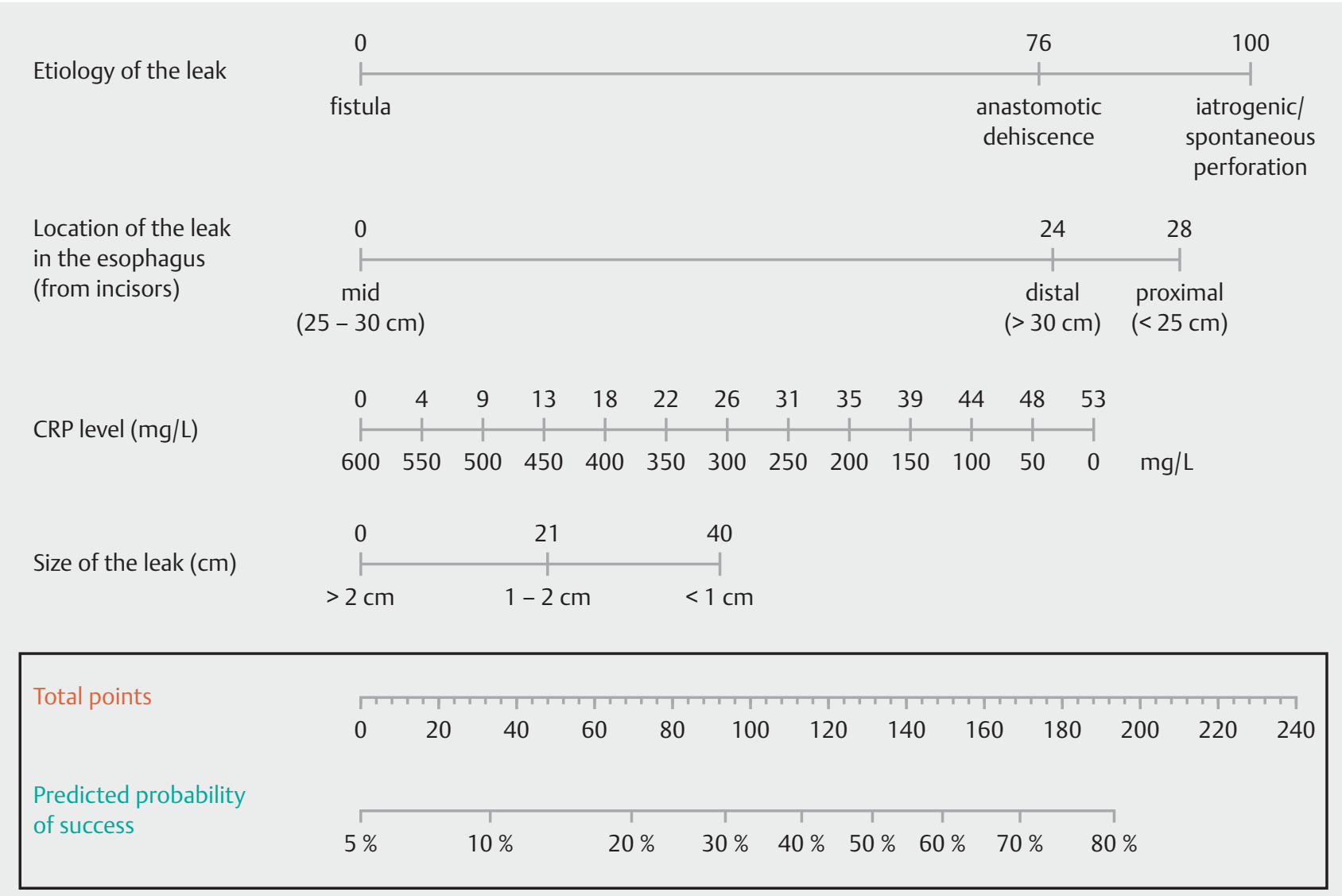

- Fig. 2 Nomogram of the prediction rule. Add up the points scored for each predictor to calculate the total number of points. The "total points" line corresponds with a "predicted probability of success" as indicated by the bottom line.

etiology of leakage and defect size were statistically significant and independent predictors of successful stent therapy in our multivariable regression model. In the literature, particularly esophago-respiratory fistulae are associated with poorer closure rates compared with anastomotic leaks and perforations $[15,16,18]$. Benign fistulae mainly develop after surgery or 

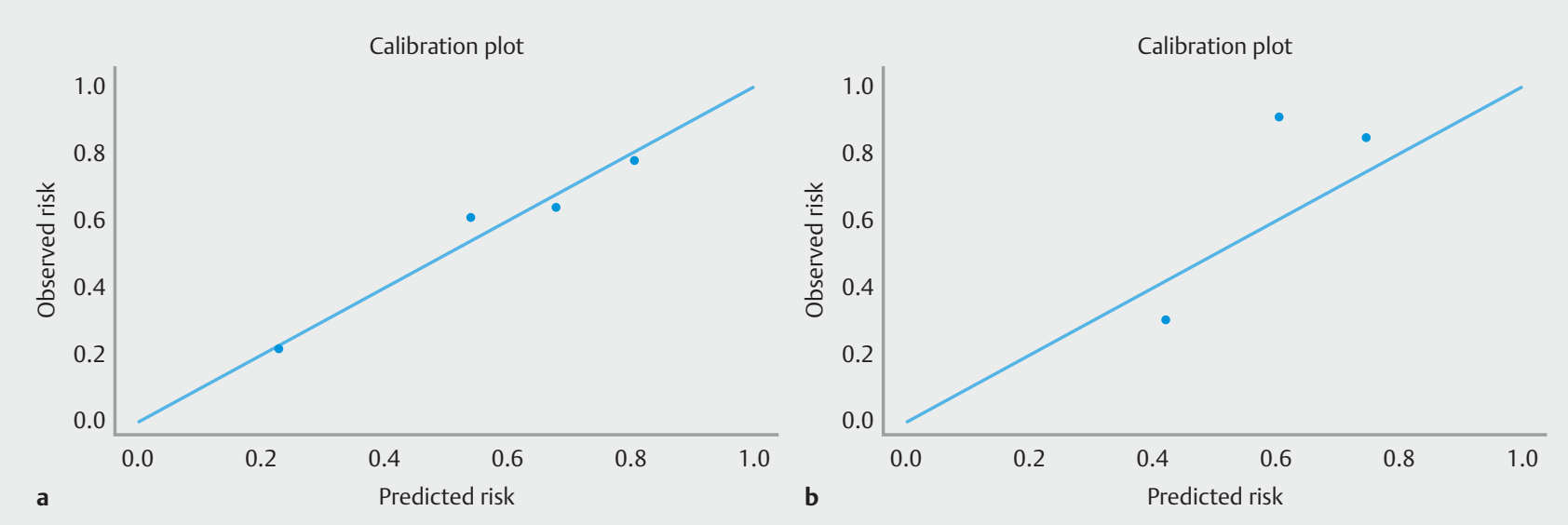

- Fig.3 Calibration plots of the predicted and observed outcomes. a Derivation sample. b Validation sample.

$\checkmark$ Table 5 Classification accuracy of the prediction rule.

\begin{tabular}{|l|l|l|}
\hline & $\begin{array}{c}\text { Derivation } \\
\text { sample (n=145) }\end{array}$ & $\begin{array}{l}\text { Validation } \\
\text { sample (n=59) }\end{array}$ \\
\hline Predicted probability of success $\geq 70 \%, \%(n / N)$ & \\
\hline - Positive predictive value & $79(38 / 48)$ & $87(13 / 15)$ \\
\hline - False positive rate & $16(10 / 64)$ & $11(2 / 19)$ \\
\hline - Sensitivity & $47(38 / 81)$ & $33(13 / 40)$ \\
\hline - Specificity & $84(54 / 64)$ & $89(17 / 19)$ \\
\hline Predicted probability of success $\leq 50 \%, \%(n / N)$ & \\
\hline - Negative predictive value & $64(32 / 50)$ & $86(12 / 14)$ \\
\hline - False negative rate & $22(18 / 81)$ & $5(2 / 40)$ \\
\hline - Sensitivity & $78(63 / 81)$ & $95(38 / 40)$ \\
\hline - Specificity & $50(32 / 64)$ & $63(12 / 19)$ \\
\hline
\end{tabular}

radiotherapy [24]. The altered and impaired vascularization status in these patients often results in a chronic and persisting defect that shows a poor tendency of healing with stent ther- apy. This is also reflected by our data, where stent therapy was successful in only $16 \%$ of patients with a fistula in the derivation sample and in none of the five patients in the validation sample. The success rate in patients with large defects $(>2 \mathrm{~cm})$ was also much lower than for patients with small defects $(<1 \mathrm{~cm})$ in both the derivation ( $46 \%$ vs. $60 \%$ ) and validation ( $41 \%$ vs. $71 \%$ ) samples. Large defects were also associated with unsuccessful closure in two studies that used a cutoff of $1.5 \mathrm{~cm}$ and $3 \mathrm{~cm} \mathrm{[17,}$ 19]. The defect size is estimated by the endoscopist during endoscopy and is therefore subject to interobserver variability. In our study, four endoscopists assessed the defect size by the available endoscopic images of the baseline procedure ( $\triangleright$ Fig. $\mathbf{4 a - c}$ ), showing fair overall interobserver agreement. Since defect size is an important predictor of success, we emphasize the importance of an accurate measurement during endoscopy. For an accurate estimation, endoscopic images of the defect should preferably be made in the presence of a reference tool, for instance the duodenal tube, which has a fixed diameter.

Previous studies have shown that septic state correlates with the success of stent therapy. Patients fulfilling severe sepsis criteria and infectious patients showed poorer success rates of stent therapy $[19,20]$. For practical reasons and clinical use, we
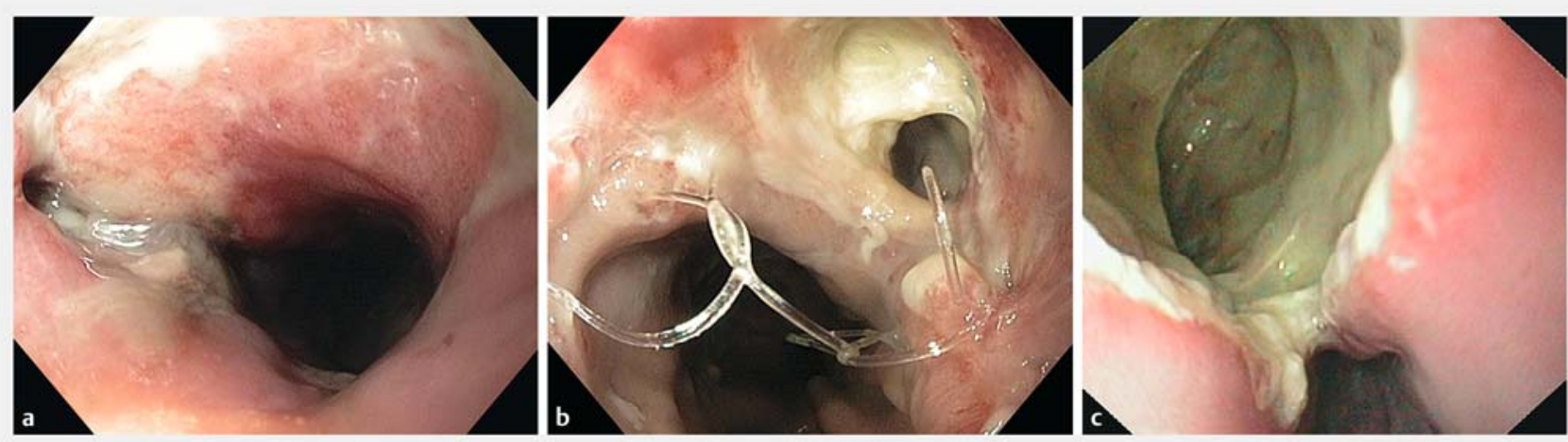

- Fig. 4 Endoscopic images of esophageal leaks at time of the baseline procedure. a, b Anastomotic dehiscence after esophagectomy. $\mathbf{c}$ Perforation after balloon dilation for stenosis after Nissen fundoplication. 
defined septic state as highest CRP level in the past 7 days before stent placement. It is likely that a CRP level within 12 hours of the stent placement procedure will have a stronger correlation with the outcome of stent therapy. However, the retrospective data in our study forced us to expand the window of CRP measurement. We furthermore included the location of the defect in the esophagus in the prediction rule. A cervical anastomosis after esophagectomy is a known risk factor for the development of anastomotic leakage $[2,25]$. This suggests a worse perfusion status than anastomoses located in the thorax. Location in the esophagus is therefore likely to affect the healing tendency of the defect.

Thoracic or cervical radiotherapy was also part of our initial, pre-specified prediction model. Although neoadjuvant chemoradiotherapy did not correlate with the development of anastomotic leaks in the literature $[25,26]$, we had included radiotherapy as a predictor of success based on the assumption that radiation injury to the esophagus and surrounding tissues may hamper wound healing due to a damaged vasculature. However, in multivariable regression analysis, the predictive value of this factor was negligible, with an estimated regression coefficient of 0.0032 and a $P$ value of 0.995 . We therefore dropped radiotherapy from the model. In the literature, the delay between the index procedure, meaning the procedure that caused the leak, and stent placement consistently impacted on the success of stent therapy. The time to stent placement was significantly shorter in patients with successful closure compared with patients in whom stent placement failed [17], and immediate stent placement resulted in higher success rates of stent therapy than in cases with a delay of more than 1 week to stent placement [18-20]. After careful consideration we chose to omit "delay to stent placement" from the model, because of the little variation, and thereby expected limited contribution to the predicted probability, and to prevent overfitting of the model.

This prediction rule is only useful when the predicted probability of success is $\geq 70 \%$ or $\leq 50 \%$, because of the high positive and negative predictive values, respectively. Predicted probabilities between $50 \%$ and $70 \%$ poorly discriminated between failure and success of stent therapy. For a predicted probability $\leq 50 \%$, we suggest to reconsider stent placement because the chance of success is between $5 \%$ and $22 \%$. For example, a patient with a small $(<1 \mathrm{~cm})$, chronic postoperative esophago-respiratory fistula in the proximal esophagus without elevated CRP levels, has a less than $50 \%$ chance of successful stent therapy according to the prediction rule. It can therefore be discussed with the patient that the defect is unlikely to heal with stent therapy. Although evidence is scarce, alternative treatment options in this case may be endoscopic clipping or surgical repair $[27,28]$. A case series including patients with small $(<2 \mathrm{~cm})$, mainly chronic, postoperative leaks and fistulae reported a closure rate of $89 \%(8 / 9)$ after over-the-scope clip placement in the upper gastrointestinal tract [29]. Endoscopic clipping has also been shown to be effective for the treatment of acute perforations caused by endoscopic procedures, with a pooled closure rate of approximately $90 \%$ [30]. In selected patients, surgical repair of benign esophago-respiratory fistulae was success- ful in $72 \%(18 / 25)$ [24]. Patients with postsurgical esophagorespiratory fistulae often had neoadjuvant chemoradiation, which hampers healing of the defect. In some patients with symptomatic fistulae who are unfit for surgery, stent placement may reduce symptoms and allow for oral intake despite a poor probability of closure of the defect. In this case, stent placement can also be used for "palliation" of symptoms.

Another example of a patient who has a less than $50 \%$ predicted probability of successful stent therapy, is a septic patient with a CRP level of $300 \mathrm{mg} / \mathrm{L}$ who is diagnosed with a large $(>2 \mathrm{~cm})$ anastomotic dehiscence in the mid esophagus after esophagectomy. Alternative options are conservative treatment with adequate drainage of infected fluid collections, or primary surgical repair of the defect [31-33]. A promising alternative for the treatment of upper gastrointestinal leaks is endoscopic vacuum therapy (E-Vac) [34]. In small retrospective studies, E-Vac showed superior closure rates compared with stent placement: $84 \%-93 \%$ vs. $54 \%-63 \%$ [35, 36]. Finally, we want to emphasize the importance of adequate abscess drainage whenever stent placement is considered.

The main limitations of our study are its retrospective design and the small validation sample. CRP level and defect size were missing in $24 \%$ and $15 \%$ of cases in the derivation sample, and in $24 \%$ and $20 \%$ of cases in the validation sample. To deal with missing values, the prediction model was estimated using a multiple imputed dataset with five rounds of imputation. To examine the performance of the model in the external validation sample, we also imputed missing values in the validation dataset. Although we have included all patients treated with stents for upper gastrointestinal leaks, this prediction model is mainly applicable to patients with esophageal leaks, because only six patients $(4 \%)$ in the derivation sample had a gastric leak.

In conclusion, using four clinical variables (i. e. etiology, location, CRP level, and size of the leak), this novel prediction rule significantly discriminated between failure and success of stent therapy for benign upper gastrointestinal leakage. Etiology fistula and defect size $>2 \mathrm{~cm}$ were independent and statistically significant predictors of stent failure. The individual predicted probability of successful stent therapy by the prediction rule can be derived from the nomogram. This prediction rule can support the physician in clinical decision-making and informing patients when the predicted probability of success is $\geq 70 \%$ or $\leq$ $50 \%$. Predicted probabilities between $50 \%$ and $70 \%$ poorly predicted the outcome of stent therapy.

\section{Competing interests}

Dr. van Berge Henegouwen is a consultant for Medtronic. Dr. Fockens has received consulting fees from Boston Scientific, Cook Medical, Fujifilm, Medtronic, and Olympus. Dr. Vleggaar has received research grants from Boston Scientific and is a consultant for Boston Scientific. Dr. Spaander has received research grants from Boston Scientific. Dr. van Hooft has received research grants from Cook Medical and Abbott, and is a consultant for Boston Scientific and Medtronic. 


\section{References}

[1] Markar S, Gronnier C, Duhamel A et al. Pattern of postoperative mortality after esophageal cancer resection according to center volume: results from a large European multicenter study. Ann Surg Oncol 2015; 22: 2615-2623

[2] Kassis ES, Kosinski AS, Ross P Jr. et al. Predictors of anastomotic leak after esophagectomy: an analysis of the society of thoracic surgeons general thoracic database. Ann Thorac Surg 2013; 96: 1919-1926

[3] Rutegard M, Lagergren P, Rouvelas I et al. Intrathoracic anastomotic leakage and mortality after esophageal cancer resection: a population-based study. Ann Surg Oncol 2012; 19: 99-103

[4] Schuchert M], Abbas G, Nason KS et al. Impact of anastomotic leak on outcomes after transhiatal esophagectomy. Surgery 2010; 148: 831 838; discussion 838-840

[5] Van Daele E, Van de Putte D, Ceelen W et al. Risk factors and consequences of anastomotic leakage after Ivor Lewis oesophagectomy. Interact Cardiovasc Thorac Surg 2016; 22: $32-37$

[6] Zehetner ], DeMeester SR, Alicuben ET et al. Intraoperative assessment of perfusion of the gastric graft and correlation with anastomotic leaks after esophagectomy. Ann Surg 2015; 262: 74- 78

[7] Raymond DP, Seder CW, Wright CD et al. Predictors of major morbidity or mortality after resection for esophageal cancer: a Society of Thoracic Surgeons General Thoracic Surgery Database risk adjustment model. Ann Thorac Surg 2016; 102: 207 - 214

[8] van der Schaaf M, Lagergren J, Lagergren P. Persisting symptoms after intrathoracic anastomotic leak following oesophagectomy for cancer. Br J Surg 2012; 99: 95 - 99

[9] Luketich JD, Pennathur A, Franchetti Y et al. Minimally invasive esophagectomy: results of a prospective phase II multicenter trial - the Eastern Cooperative Oncology Group (E2202) study. Ann Surg 2015; 261: $702-707$

[10] Kataoka K, Takeuchi H, Mizusawa J et al. Prognostic impact of postoperative morbidity after esophagectomy for esophageal cancer: exploratory analysis of JCOG9907. Ann Surgery 2017; 265: 1152-1157

[11] Goense L, van Rossum PS, Weijs TJ et al. Aortic calcification increases the risk of anastomotic leakage after Ivor-Lewis esophagectomy. Ann Thorac Surg 2016; 102: 247-252

[12] Deldycke A, Van Daele E, Ceelen W et al. Functional outcome after Ivor Lewis esophagectomy for cancer. J Surg Oncol 2016; 113: 24-28

[13] Fischer C, Lingsma H, Hardwick R et al. Risk adjustment models for short-term outcomes after surgical resection for oesophagogastric cancer. Br J Surg 2016; 103: 105-116

[14] Dasari BV, Neely D, Kennedy A et al. The role of esophageal stents in the management of esophageal anastomotic leaks and benign esophageal perforations. Ann Surg 2014; 259: 852-860

[15] van Halsema EE, van Hooft JE. Clinical outcomes of self-expandable stent placement for benign esophageal diseases: a pooled analysis of the literature. World J Gastrointest Endosc 2015; 7: 135-153

[16] Persson S, Rouvelas I, Kumagai K et al. Treatment of esophageal anastomotic leakage with self-expanding metal stents: analysis of risk factors for treatment failure. Endosc Int Open 2016; 4: E420 - 426

[17] El Hajj II, Imperiale TF, Rex DK et al. Treatment of esophageal leaks, fistulae, and perforations with temporary stents: evaluation of efficacy, adverse events, and factors associated with successful outcomes. Gastrointest Endosc 2014; 79: 589- 598

[18] Gubler C, Bauerfeind P. Self-expandable stents for benign esophageal leakages and perforations: long-term single-center experience. Scand J Gastroenterol 2014; 49: 23-29
[19] Orive-Calzada A, Calderon-Garcia A, Bernal-Martinez A et al. Closure of benign leaks, perforations, and fistulas with temporary placement of fully covered metal stents: a retrospective analysis. Surg Laparosc Endosc Percutan Tech 2014; 24: 528 - 536

[20] Swinnen J, Eisendrath P, Rigaux J et al. Self-expandable metal stents for the treatment of benign upper Gl leaks and perforations. Gastrointest Endosc 2011; 73: 890-899

[21] Hirdes MM, Siersema PD, Houben MH et al. Stent-in-stent technique for removal of embedded esophageal self-expanding metal stents. Am J Gastroenterol 2011; 106: 286-293

[22] van Buuren S, Groothuis-Oudshoorn K. mice: Multivariate Imputation by Chained Equations in R. J Stat Soft 2011; 45: 1 - 6

[23] Steyerberg EW, Eijkemans MJ, Harrell FEJr. et al. Prognostic modelling with logistic regression analysis: a comparison of selection and estimation methods in small data sets. Stat Med 2000; 19: 1059-1079

[24] Lenz C], Bick BL, Katzka D et al. Esophagorespiratory fistulas: survival and outcomes of treatment. J Clin Gastroenterol 2016: doi:10.1097| MCG. 0000000000000751

[25] Gronnier C, Trechot B, Duhamel A et al. Impact of neoadjuvant chemoradiotherapy on postoperative outcomes after esophageal cancer resection: results of a European multicenter study. Ann Surg 2014; 260: 764 - 770; discussion 770-761

[26] van Hagen P, Hulshof MC, van Lanschot JJ et al. Preoperative chemoradiotherapy for esophageal or junctional cancer. New Engl J Med 2012; 366: 2074-2084

[27] Gonzalez JM, Servajean C, Aider B et al. Efficacy of the endoscopic management of postoperative fistulas of leakages after esophageal surgery for cancer: a retrospective series. Surg Endosc 2016; 30: $4895-4903$

[28] Silon B, Siddiqui AA, Taylor L] et al. Endoscopic management of esophagorespiratory fistulas: a multicenter retrospective study of techniques and outcomes. Dig Dis Sci 2017; 62: 424-431

[29] Mennigen R, Colombo-Benkmann M, Senninger $N$ et al. Endoscopic closure of postoperative gastrointestinal leakages and fistulas with the Over-the-Scope Clip (OTSC). J Gastrointest Surg 2013; 17: 1058 1065

[30] Verlaan T, Voermans RP, van Berge Henegouwen Ml et al. Endoscopic closure of acute perforations of the GI tract: a systematic review of the literature. Gastrointest Endosc 2015; 82: 618-628 e615

[31] Guo ], Chu X, Liu Y et al. Choice of therapeutic strategies in intrathoracic anastomotic leak following esophagectomy. World J Surg Oncol 2014; 12: 402

[32] Lee DH, Kim HR, Kim SR et al. Comparison of clinical outcomes after conservative and surgical treatment of isolated anastomotic leaks after esophagectomy for esophageal cancer. Dis Esophagus 2013; 26: $609-615$

[33] Lee S, Ahn JY, Jung HY et al. Clinical outcomes of postoperative upper gastrointestinal leakage according to treatment modality. Dig Dis Sci 2016; 61: $523-532$

[34] Leeds SG, Burdick JS, Fleshman JW. Endoluminal vacuum therapy for esophageal and upper intestinal anastomotic leaks. JAMA Surg 2016; 151: $573-574$

[35] Brangewitz M, Voigtlander T, Helfritz FA et al. Endoscopic closure of esophageal intrathoracic leaks: stent versus endoscopic vacuum-assisted closure, a retrospective analysis. Endoscopy 2013; 45: 433 438

[36] Mennigen R, Harting C, Lindner K et al. Comparison of endoscopic vacuum therapy versus stent for anastomotic leak after esophagectomy. J Gastrointest Surg 2015; 19: 1229-1235 